International Journal of Health Sciences
Available online at www.sciencescholar.us
Vol. 5 No. 3, December 2021, pages: $393-402$
e-ISSN: 2550-696X, p-ISSN: 2550-6978
https://doi.org/10.53730/ijhs.v5n3.1698

\title{
Kidney Complications after COVID-19
}

\author{
CrossMark \\ Sanobar Rahkmonova a , Jurabek Khaytboyev b, Nargiza Zargarova c, Dilfuza Otajonova d,
Khabibulla Kazakov e
}

Manuscript submitted: 09 May 2021, Manuscript revised: 18 August 2021, Accepted for publication: 27 September 2021

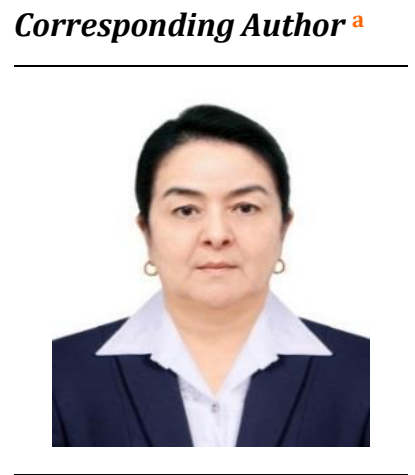

Keywords

COVID-19;

ferritin;

health problems;

health systems;

healthy cells;

healthy lifestyle;

metabolism;

patient health;

\begin{abstract}
This article discusses renal complications after COVID-19. It has now become clear that infection with the SARS-Cov-2 virus, which causes COVID-19, is almost identical - and this tells scientists which organs are most at risk. Specifically, the respiratory tract, intestines, and, most likely, the kidneys, since it is there that both proteins necessary for the virus are present. Even the Ebola virus, found in the eye fluid of ex-patients months after recovery, causes blindness in 40 percent of those infected later. Therefore, given that SARS-CoV2 is more likely to damage the lungs, doctors have speculated that the virus may cause irreversible changes in the respiratory system.
\end{abstract}

\section{Contents}

Abstract

International Journal of Health Sciences (c) 2021. This is an open access article under the CC BY-NC-ND license (https://creativecommons.org/licenses/by-nc-nd/4.0/).

Materials and Methods

3 Results and Discussions

4 Conclusion.

Acknowledgments..

References

Biography of Authors.

a Urgench Branch of the Medical Academy, Urgench, Uzbekistan

${ }^{\text {b }}$ Urgench Branch of the Medical Academy, Urgench, Uzbekistan

c Urgench Branch of the Medical Academy, Urgench, Uzbekistan

d Urgench Branch of the Medical Academy, Urgench, Uzbekistan

e Urgench Branch of the Medical Academy, Urgench, Uzbekistan 


\section{Introduction}

The COVID-19 pandemic is a pandemic of COVID-19 coronavirus infection caused by SARS-CoV-2, a severe coronavirus of acute respiratory syndrome (Martino et al., 2020). The disease, first reported in December 2019 in Wuhan, China, was declared a pandemic by the World Health Organization on March 11, 2020. Experts investigating the origin of the coronavirus have ruled out the assumption that the disease originated in a laboratory (Shingare et al., 2020). The data, which formed the basis of the research process, were collected as a result of joint research by Chinese and foreign scientists. It was noted that the delay in the publication of the conclusions was not due to possible disagreements between the experts. As of September 1, 2021, the virus has infected more than 218 million people in more than 192 countries and territories; More than 4,520,000 people died of the disease, and more than 195 million were cured.

The virus is transmitted from person to person through droplets released during coughing and coughing, similar to the flu. Although the virus is most contagious when a patient develops symptoms, it can also be transmitted to others before symptoms appear. Symptoms usually appear within five days, but this period can last from 2 to 14 days (Pei et al., 2020). The main symptoms of the disease are fever, cough, and shortness of breath.

\section{Materials and Methods}

The disease can cause pneumonia and acute respiratory distress syndrome. There is currently no vaccine or drug against COVID-19 (Hassanzadeh et al., 2022). Disease management consists of symptomatic treatment and adjuvant therapy. To prevent the spread of the disease, it is recommended to wash hands, cover the mouth when coughing, and stay away from others (especially those who do not feel well). Individuals suspected of being infected with the virus are advised to isolate themselves for 14 days (Liu et al., 2021).

Measures were taken to prevent the spread of the disease, such as travel restrictions, quarantine, curfews, deferrals and cancellations, and closures. In particular, the province of Hubei, where the virus began to spread, was quarantined, quarantined in Italy, Spain, France, the Czech Republic, and Germany, curfews were imposed in China and South Korea, borders were closed in various countries or restrictions were imposed on tourists, airports and train stations. Control measures have been put in place, and recommendations have been issued against travel to areas where the virus is endemic. In at least 115 countries, universities and schools were closed masse or in some areas, affecting more than 950 million students. The first case of the virus in Uzbekistan was reported on March 15. The pandemic has caused many socio-economic challenges globally, led to the postponement or cancellation of sporting and cultural events, and raised concerns about shortages of medicines, electronics, and food (Lin et al., 2020). There has also been a flurry of misinformation and conspiracy theories about viruses and pandemics on the Internet, xenophobia, and racism in various countries, and panicked shoppers buying large quantities of goods such as toilet paper. In July 2021, WHO President Tedros Adhanom Ghebreyesus said that vaccinations could lead to a vaccine-resistant strain of the coronavirus.

Infections and medications can also have a negative effect on the condition of the body. In addition to the general changes specific to the disease in people cured of COVID-19, it was found that individual injuries are also observed in the body of each patient (Craig-Schapiro et al., 2021). Patient health due to water should be closely monitored (Löwe et al., 2004). Laboratory tests help to assess the functional status of organs in the human body after the disease and to identify complications (Löwe et al., 2010). Patients recovering from COVID-19 should focus on 3 systems that are most likely to be injured: lung, kidney, and brain function (Adamsick et al., 2020).

If the patient has symptoms of shortness of breath lasting more than two months and does not decrease in dynamic follow-up, a cardiologist's consultation and cardiac UTT examination should also be performed. Of the tests, a complete blood count is the fastest, simplest, and most informative test. This test focuses primarily on the detection of eosinophils that exhibit an allergic component (Alasfar \& Avery, 2020). At the same time, 
the state of the inflammatory process in the body (leukocytes, lymphocytes, e CHT) and anemia, a common complication, are also assessed.

Pseudomembranous colitis is one of the most common complications in patients receiving antibacterial therapy for a long time. This complication is observed in patients with unexplained diarrhea, persistent pain in the abdomen. In these cases, a deeper examination of the patient, the need for narrow specialist advice.

Signs of inflammation:

interpreted by total blood test + e CHT, leukocyte formula, S-reactive protein, ferritin.

Blood coagulation system:

- D-dimer, prothrombin, fibrinogen;

- Assess the saturation of the body with vitamin D:

- 25-OH vitamin D;

Kidney status:

- Creatinine, urea, protein and carbohydrate metabolism, glucose, albumin, cystatin S;

Timely detection of autoimmune processes:

- Antibodies to nuclear antigens are performed through screening analyzes.

To support a healthy lifestyle and increase physical activity, the general population and the formation of a healthy lifestyle in every household, the fight against harmful habits, the development of medical culture, physical education, sports, and nutrition should be actively promoted and followed (Pronk et al., 2004). Data from British researchers suggest that the first symptoms of the disease may appear, disappear and recur within 30 or more days, which is significantly more than the official two-week period set by WHO experts. For some, the disease may be the beginning of a long and tragic battle against the virus, which could turn into a new "postcoronavirus syndrome" (Loef \& Walach, 2012; Widjaja, 2021). According to the prestigious journal Science, this type of coronavirus "does things that no human has ever known" (Lentine et al., 2020; Widana et al., 2021).

As officials took steps to stop the spread of the infection, and as doctors searched (in vain) for a cure or vaccine for the disease, we learned new things about the coronavirus, and perceptions about it changed. At first, it seemed to be a common respiratory disease like SARS or bird flu, but later it became clear that the coronavirus (its official name is SARS-CoV-2) infects the lungs, brain, nasopharynx, eyes, heart, blood vessels, has been shown to affect the liver, kidneys, and intestines, i.e., virtually all vital organs (Kunutsor \& Laukkanen, 2020). Studies to date have shown that COVID-19 has a variety of symptoms, and for those with a more severe form of the disease, the long-term consequences can be serious: from lung tissue depletion and inflammation of the heart muscle to kidney failure, arrhythmia, liver injury, cognitive impairment, psychosis with sudden mood swings, etc.

The effects of the disease on humans are yet to be determined, but the effects of the disease continue to be felt even by those suffering from its milder form. Even the Ebola virus, found in the eye fluid of ex-patients months after recovery, causes blindness in 40 percent of those infected later. Therefore, given that SARS-CoV2 is more likely to damage the lungs, doctors have speculated that the virus may cause irreversible changes in the respiratory system. Experts from the Wuhan University of Technology reported in March that computed tomography scans of 66 of the 70 patients who had experienced pneumonia caused by COVID-19 showed lung damage. In some patients, lung function may not be fully restored (Yarijani \& Najafi, 2021).

These injuries range from obstruction of blood vessels in the alveoli to scarring of the lung tissue. These scars or thickening of the tissue is called pulmonary fibrosis and causes shortness of breath. There is currently no way to stop or reverse this process (Lim et al., 2020). "It's true that fibrosis can stabilize and not change over time. In some patients, lung function is not fully restored, but the consequences are not so great. But there are also cases of developing fibrosis, which is very dangerous and can lead to death sooner or later," said Louise Wayne, a professor at the University of Leicester. In addition, before the introduction of strict quarantine in the UK, a professional medical organization specializing in the training of British resuscitators the Faculty of Intensive Care Medicine (FICM) - took up to 15 years for lung recovery in patients experiencing

Rahkmonova, S., Khaytboyev, J., Zargarova, N., Otajonova, D., \& Kazakov, K. (2021). Kidney complications after COVID-19. International Journal of Health Sciences, 5(3), 393-402. https://doi.org/10.53730/ijhs.v5n3.1698 
severe forms of COVID-19 had been warned that he could be seriously injured as needed. The FICM noted that the majority of patients admitted to the intensive care unit developed acute respiratory distress syndrome (ARDS) - the most severe inflammation of the lungs, in which fluid enters the alveoli and results in shortness of breath without special equipment. it becomes impossible to get. Given the history of lung tissue injury in patients with SARS and MERS, a medical team led by Melina Husseini, a radiologist at the University of California, Los Angeles, monitored COVID-19 patients and assessed their lungs for "long-term or permanent damage, including to assess fibrosis" (Post et al., 2020; Attamimi et al., 2020).

Although the coronavirus primarily infects the lungs, in some patients the infection has spread to the kidneys. A sample study in China found that 27 percent of the 85 patients admitted to Wuhan hospitals with coronavirus had kidney problems. Another study found that in the urine of nearly 200 hospitalized patients in Hubei and Sichuan provinces, a protein that confirmed the infection was found in 59 percent of cases and blood in 44 percent of cases indicating severe kidney damage (Han \& Ye, 2021). In addition, patients with acute renal failure (ARF) had a 5-fold higher risk of death than normal patients with coronavirus.

Recently, however, doctors in the United States and China have noted that the number of people with renal failure has sharply increased among those infected. As a result, hospitals are already experiencing an acute shortage of hemodialysis machines ("artificial kidneys"), as well as medical staff who can handle such patients, not to mention the necessary drugs. Since the new virus is still extremely poorly understood, it cannot be said with certainty whether it affects the kidneys directly or if it is just a side effect of infection. It is known that people who already have health problems and comorbidities tend to tolerate COVID-19 more severely than others. Many patients may simply not be aware that they have kidney problems (Gubler, 2002; Spoorthy et al., 2020). But the matter may be in the excessive activation of the immune system: upon discovering an unfamiliar infection, it begins to attack not only the virus itself but also the healthy cells of its own body, including such a sensitive organ as the kidneys (Waleka et al., 2020; Metaxa et al., 2014).

According to one of the versions, the infected are more likely to develop blood clots in the vessels, and this, in turn, can also adversely affect the kidneys, penetrated by a network of thinnest capillaries. It is also known that the kidneys are closely related to the liver and bladder. And now many doctors fear that if patients with COVID-19 have kidney problems, then shortly a wave of complications may begin on the organs associated with them (Chan et al., 2021). At the moment, these are all just theories, although, as the experience of several previous epidemics shows, health workers should prepare for the most unexpected options for the development of infection.

Already now, in many hospitals, doctors have to decide which of the patients to connect to hemodialysis machines and in what order, since there are not enough "artificial kidneys" for all. In other words, only those who have a higher chance of survival have to be saved - the more severely ill may have to die without the necessary help. The situation is further exacerbated by the acute shortage of trained hemodialysis nurses.

According to the latest data from American doctors, from 20 to $40 \%$ of all patients admitted to intensive care with coronavirus suffer from serious kidney dysfunction - and all of them need hemodialysis, writes the New York Times (Toapanta et al., 2021). This has already led to a shortage of both the "artificial kidney" devices themselves and the medicines necessary for this procedure since no one expected such a sharp increase in the number of patients requiring this procedure. According to Dr. Barbara Murphy of the Mount Sinai US hospital chain, the number of patients in dire need of hemodialysis has tripled in just one clinic.

In the United States, artificial kidney devices and all the materials necessary for them are produced by only two large companies. Both report that the demand for their products has grown fivefold - not only in America but also in China and European countries. In some hospitals in New York, doctors are forced to resort to a less effective and more risky method of blood purification - peritoneal dialysis. It is usually used for milder kidney problems - but doctors say it's better than nothing. In the early 2000s, the SARS epidemic of "atypical pneumonia" spread to three dozen countries. Then the scientists found out that the causative agent of the disease, the SARS-Co V virus, enters the cells of the human body with the help of two proteins: one is the cellular receptor ACE2, the other is the TMPRSS2 enzyme.

It has now become clear that infection with the SARS-Cov-2 virus, which causes COVID-19, is almost identical - and this tells scientists which organs are most at risk. Specifically, the respiratory tract, intestines, and, most likely, the kidneys, since it is there that both proteins necessary for the virus are present (Hussin et al., 2021). In addition, health systems in many countries have drawn lessons from the HIV epidemic that began in the early 1980s (Travis et al., 2004; Samb et al., 2010). Then they also said that the disease poses a danger 
only to representatives of certain risk groups, and for a long time it was believed that HIV threatens only homosexual men, as well as those who have undergone a blood transfusion or have Haitian origin.

But after AIDS began to die, including white heterosexual wealthy women, the authorities became agitated and urgently changed the rules for testing new drugs and medicines - so that in extreme situations like an epidemic this could be done quickly, literally in months, not years. Roughly the same is happening now. Quarantine is being introduced almost everywhere, and huge forces are being thrown into the search for a vaccine and specific drugs for the new coronavirus. Human testing of some prototype vaccines has already begun. But the development of a vaccine, and even more so a coronavirus cure, can take a very long time. It is possible that over time, COVID-19 will become just a chronic disease that can be controlled (Bocheliuk et al., 2021).

Acute kidney damage is one of the deaths from COVID-19, especially in severe cases, she said. At the same time, the nephrologist added, patients may not know that they have kidney disease, since they did not notice anything before, and the coronavirus can sharply exacerbate processes that had previously developed slowly. Anna Korobkina listed what needs to be done for patients who are being treated for COVID-19 at home to identify impaired kidney function.

"Get a general urine test for protein, a biochemical blood test for creatinine levels. This is a basic screening to check how well your kidneys are working. Subsequently, to exclude the effect of coronavirus on the renal tissue, it is necessary to monitor the blood clotting parameters and to prevent thrombosis, because blood clots are formed in the renal vessels as well," Anna Korobkina recommended in an interview with Sputnik radio. They conducted a study in which 1.6 thousand patients with acute kidney injury took part (Ahmadian et al., 2021). Specialists monitored people who were diagnosed with pathology during their hospital stay with coronavirus, as well as those who did not have this infection.

\section{Results and Discussions}

According to American scientists, $24-57 \%$ of hospitalizations with COVID-19 and from $61 \%$ to $78 \%$ of hospitalizations in intensive care units are accompanied by kidney damage. Doctors tracked the condition of the patients 21 days after discharge and it turned out that those who underwent coronavirus did not recover their original kidney function. Moreover, they were more likely to require dialysis than non-COVID-19 patients with acute kidney inflammation (Aleebrahim-Dehkordi et al., 2020). Also, those who underwent coronavirus had a higher risk of developing chronic renal failure (CRF), Izvestia writes. As noted by urologist Artur Tedeev, the available data indicate a direct effect of the virus on the kidney tissue. It can cause the development of acute renal failure, which is microscopically defined as severe acute tubular necrosis.

Most often, such a complication develops in people with cardiovascular pathologies (hypertension, coronary heart disease) and diabetes mellitus, added Tedeev. The kidneys, the author of the material emphasizes, are organs vulnerable to coronavirus. This is because there are twice as many receptors in the form of which the virus enters the body than, for example, in the heart or esophagus. Also, doctor Anna Korobkina notes that serious kidney damage is one of the factors leading to death with COVID.

The main thing to do when diagnosing coronavirus is to donate blood and urine. It is necessary to check the presence of the level of creatinine in the blood, as well as the presence of protein in the urine. This will let you know if your kidneys are working properly. And so that the coronavirus cannot damage the kidneys, the expert explains, it is necessary to monitor blood clotting and the formation of blood clots. The transferred coronavirus can affect the kidneys and these consequences can persist for life, causing serious damage to the functioning of this organ, reports The New York Times, citing a study by a group of American scientists from St. Louis (Kunutsor \& Laukkanen, 2020). The study involved 89.2 thousand people who were diagnosed with COVID-19 from March 1, 2020, to March 15, 2021. Scientists also used data from 1.6 million people who did not suffer from COVID-19.

As a result, in the period from one to six months after infection, people with COVID-19 were more susceptible to decreased kidney function (by 35\%) than those in whom the coronavirus was not detected. "People who survive the first 30 days of COVID are at risk of developing kidney failure," the newspaper quoted Ziyad Al-Ali, a nephrologist and assistant professor at the University of Washington School of Medicine, who co-authored the study. He also noted that in 4.7 thousand study participants who underwent COVID-19,

Rahkmonova, S., Khaytboyev, J., Zargarova, N., Otajonova, D., \& Kazakov, K. (2021). Kidney complications after COVID-19. International Journal of Health Sciences, 5(3), 393-402. https://doi.org/10.53730/ijhs.v5n3.1698 
kidney function decreased by $30 \%$ during the year. This is equivalent to roughly 30 years of "aging" of the organ, said Perry Wilson, assistant professor of medicine at Yale, who was not involved in the study.

In 220 patients involved in the study, scientists identified the last stage of kidney disease, when $85 \%$ of their functions are lost. At the beginning of the pandemic, Russia's chief nephrologist Yevgeny Shilov warned that coronavirus is extremely dangerous for those suffering from chronic kidney disease. He explained that such patients have a higher risk of not only infection but also death. According to the expert, the causes of chronic kidney disease are, for example, diabetes mellitus, hypertension, atherosclerosis, and other diseases, which in themselves contribute to high morbidity and complications. Six months later, British scientists concluded that kidney disease more than doubles the risk of death from coronavirus infection.

COVID-19 disease can affect kidney function. Nephrologist Anna Korobkina, in an interview with Sputnik Radio, told how you can avoid the corresponding complications after the coronavirus. As the doctor noted, the virus attacks all internal organs, where there is an ACE2 enzyme - it is used as a receptor to enter the cell. These receptors are found in the bladder, esophagus, ileum, and heart. However, the kidneys are the most vulnerable organ in this sense.

It is kidney damage that is one of the most significant mortality factors in coronavirus. Moreover, the situation is complicated by kidney disease, which the person had never even suspected of - COVID-19 dramatically speeds up the processes. According to the expert, patients with coronavirus should be tested as soon as possible to identify possible kidney problems. This is a general urine test for protein and a biochemical blood test for creatinine levels. Next, you need to monitor the blood clotting parameters and prevent thrombosis, since they are often formed in the renal vessels.

1.6 thousand patients took part in the study of American specialists. Some of them were diagnosed with acute kidney damage during their hospital stay with coronavirus, while others did not tolerate this infection at all. According to experts, $24-57 \%$ of patients with COVID-19 and from $61 \%$ to $78 \%$ of those hospitalized in intensive care units have kidney damage. Doctors monitored the condition of patients 21 days after discharge and found that in those who had undergone coronavirus, the original kidney function did not recover. Moreover, they were more likely to need dialysis than non-COVID-19 patients with acute kidney inflammation.

Also, COVID-19 patients have a higher risk of developing chronic renal failure. According to the study authors, patients who have identified predictors of acute kidney damage associated with COVID-19 need close outpatient monitoring during a pandemic. Artur Tedeev, the urologist at the Medsi Clinical Diagnostic Center, confirmed that the available data indicate a direct effect of the virus on the kidney tissue. "It can cause acute renal failure, which is microscopically defined as severe acute tubular necrosis. Most often, this complication develops in people with cardiovascular pathologies (hypertension, coronary heart disease) and diabetes mellitus" he said.

As previously reported, acute kidney injury (AKI) due to infection with SARS and MERS-CoV developed in $5-15 \%$ of cases and had a high (60-90\%) mortality rate. Early reports suggested a lower incidence (3-9\%) of AKI in individuals with COVID-19 infection. Recent reports, however, have shown a higher incidence of renal impairment. A study of 59 patients with COVID-19 showed that $34 \%$ of patients had massive albuminuria on the first day of hospitalization, $63 \%$ of patients had proteinuria during the entire hospitalization. The blood urea level was increased overall by $27 \%$; elevated urea levels were also observed in $2 / 3$ of the patients who died. Computed tomography of the kidneys showed a decrease in density, which indicated inflammation and edema. Cheng et al. recently reported that among 710 consecutive hospitalized COVID-19 patients, 44\% had proteinuria and hematuria and $26.7 \%$ had hematuria upon admission. The prevalence of elevated serum creatinine and urea was $15.5 \%$ and $14.1 \%$, respectively. AKI was an independent risk factor for in-hospital mortality.

The actual mechanism of kidney damage is unclear: postulated mechanisms include sepsis leading to cytokine storm syndrome or direct cell damage by the virus. Angiotensin-converting enzyme and dipeptidyl peptidase-4, both expressed on renal tubule cells, have been identified as binding partners for SARS-CoV and MERS-CoV, respectively. Viral RNA has been identified in kidney tissue and urine in both infections (Massie et al., 2020). Recently, the Zgong laboratory in Guangzhou successfully isolated SARS-CoV-2 from an infected patient's urine sample, suggesting that the kidney is a target for this new coronavirus. 


\section{Conclusion}

Thus, COVID-19, the disease caused by the new coronavirus, poses a serious global threat to humans, which could escalate into a pandemic. It appears that kidney damage is common with this infection, and AKI is an independent predictor of mortality. The impact of this infection on patients with chronic kidney disease has not been studied, and dialysis patients suspected of having COVID-19 should be managed according to strict protocols to minimize the risk of transmission to other patients and the medical staff caring for these patients.

Acknowledgments

We are grateful to two anonymous reviewers for their valuable comments on the earlier version of this paper.

Rahkmonova, S., Khaytboyev, J., Zargarova, N., Otajonova, D., \& Kazakov, K. (2021). Kidney complications after COVID-19. International Journal of Health Sciences, 5(3), 393-402. https://doi.org/10.53730/ijhs.v5n3.1698 


\section{References}

Adamsick, M. L., Gandhi, R. G., Bidell, M. R., Elshaboury, R. H., Bhattacharyya, R. P., Kim, A. Y., ... \& Sise, M. E. (2020). Remdesivir in patients with acute or chronic kidney disease and COVID-19. Journal of the American Society of Nephrology, 31(7), 1384-1386.

Ahmadian, E., Hosseiniyan Khatibi, S. M., Razi Soofiyani, S., Abediazar, S., Shoja, M. M., Ardalan, M., \& Zununi Vahed, S. (2021). Covid-19 and kidney injury: Pathophysiology and molecular mechanisms. Reviews in medical virology, 31(3), e2176.

Alasfar, S., \& Avery, R. K. (2020). The impact of COVID-19 on kidney transplantation. Nature Reviews Nephrology, 16(10), 568-569.

Aleebrahim-Dehkordi, E., Reyhanian, A., Saberianpour, S., \& Hasanpour-Dehkordi, A. (2020). Acute kidney injury in COVID-19; a review on current knowledge. Journal of Nephropathology, 9(4), e31-e31.

Attamimi, H. R. ., Lestari, Y. ., Situmorang, B. . H. L. ., Antari, G. Y. ., \& Nugrawati, N. . (2020). Application of habituation method in germas interventionsin: the pandemic time COVID-19. International Journal of Health \& Medical Sciences, 3(1), 98-104.

Bocheliuk, V. Y., Spytska, L. V., Mamicheva, O. V., Panov, M. S., \& Kordonets, V. V. (2021). Psychological features of post-COVID syndrome course. International Journal of Health Sciences, 5(3), 276-285.

Chan, K. W., Hung, I. F. N., Tsang, O. T. Y., Wu, T. C., Tso, E. Y. K., Lung, K. C., ... \& Tang, S. C. W. (2021). Mass screening is associated with low rates of acute kidney injury among COVID-19 patients in Hong Kong. American Journal of Nephrology, 52(2), 161-172.

Craig-Schapiro, R., Salinas, T., Lubetzky, M., Abel, B. T., Sultan, S., Lee, J. R., ... \& Dadhania, D. M. (2021). COVID-19 outcomes in patients waitlisted for kidney transplantation and kidney transplant recipients. American Journal of Transplantation, 21(4), 1576-1585.

Gubler, D. J. (2002). The global emergence/resurgence of arboviral diseases as public health problems. Archives of medical research, 33(4), 330-342. https://doi.org/10.1016/S0188-4409(02)00378-8

Han, X., \& Ye, Q. (2021). Kidney involvement in COVID-19 and its treatments. Journal of medical virology, 93(3), 1387-1395.

Hassanzadeh, S., Djamali, A., Mostafavi, L., \& Pezeshgi, A. (2022). Kidney complications following COVID-19 vaccination; a review of the literature. Journal of Nephropharmacology, 11(1).

Hussin, D. A., Samah, M. A. A., Suhaimi, A. A., \& Kamarudin, M. K. A. (2021). A study on knowledge, attitude and practice of COVID-19 pandemic among the residents. International Journal of Health Sciences, 5(2), 177188.

Kunutsor, S. K., \& Laukkanen, J. A. (2020). Renal complications in COVID-19: a systematic review and metaanalysis. Annals of medicine, 52(7), 345-353.

Lentine, K. L., Vest, L. S., Schnitzler, M. A., Mannon, R. B., Kumar, V., Doshi, M. D., ... \& Axelrod, D. A. (2020). Survey of US living kidney donation and transplantation practices in the COVID-19 era. Kidney international reports, 5(11), 1894-1905. https://doi.org/10.1016/j.ekir.2020.08.017

Lim, M. A., Pranata, R., Huang, I., Yonas, E., Soeroto, A. Y., \& Supriyadi, R. (2020). Multiorgan failure with emphasis on acute kidney injury and severity of COVID-19: systematic review and meta-analysis. Canadian journal of kidney health and disease, 7, 2054358120938573.

Lin, L., Wang, X., Ren, J., Sun, Y., Yu, R., Li, K., ... \& Yang, J. (2020). Risk factors and prognosis for COVID-19induced acute kidney injury: a meta-analysis. BMJ open, 10(11), e042573.

Liu, Y. F., Zhang, Z., Pan, X. L., Xing, G. L., Zhang, Y., Liu, Z. S., \& Tu, S. H. (2021). The chronic kidney disease and acute kidney injury involvement in COVID-19 pandemic: A systematic review and meta-analysis. PloS one, 16(1), e0244779.

Liu, Y. M., Xie, J., Chen, M. M., Zhang, X., Cheng, X., Li, H., ... \& Li, H. (2021). Kidney function indicators predict adverse outcomes of COVID-19. Med, 2(1), 38-48.

Loef, M., \& Walach, H. (2012). The combined effects of healthy lifestyle behaviors on all cause mortality: a systematic review and meta-analysis. Preventive medicine, 55(3), $163-170$. https://doi.org/10.1016/j.ypmed.2012.06.017

Löwe, B., Kroenke, K., Herzog, W., \& Gräfe, K. (2004). Measuring depression outcome with a brief self-report instrument: sensitivity to change of the Patient Health Questionnaire (PHQ-9). Journal of affective disorders, 81(1), 61-66. https://doi.org/10.1016/S0165-0327(03)00198-8 
Löwe, B., Wahl, I., Rose, M., Spitzer, C., Glaesmer, H., Wingenfeld, K., ... \& Brähler, E. (2010). A 4-item measure of depression and anxiety: validation and standardization of the Patient Health Questionnaire-4 (PHQ-4) in the general population. Journal of affective disorders, 122(1-2), 86-95. https://doi.org/10.1016/j.jad.2009.06.019

Martino, F., Plebani, M., \& Ronco, C. (2020). Kidney transplant programmes during the COVID-19 pandemic. The Lancet. Respiratory Medicine, 8(5), e39.

Massie, A. B., Boyarsky, B. J., Werbel, W. A., Bae, S., Chow, E. K., Avery, R. K., ... \& Segev, D. L. (2020). Identifying scenarios of benefit or harm from kidney transplantation during the COVID-19 pandemic: a stochastic simulation and machine learning study. American Journal of Transplantation, 20(11), 2997-3007.

Metaxa, A. F., Efthimiadou, E. K., \& Kordas, G. (2014). Cellulose-based drug carriers for cancer therapy: cytotoxic evaluation in cancer and healthy cells. Materials Letters, 132, 432-435. https://doi.org/10.1016/j.matlet.2014.06.134

Pei, G., Zhang, Z., Peng, J., Liu, L., Zhang, C., Yu, C., ... \& Xu, G. (2020). Renal involvement and early prognosis in patients with COVID-19 pneumonia. Journal of the American Society of Nephrology, 31(6), 1157-1165.

Post, A., den Deurwaarder, E. S., Bakker, S. J., de Haas, R. J., van Meurs, M., Gansevoort, R. T., \& Berger, S. P. (2020). Kidney infarction in patients with COVID-19. American Journal of Kidney Diseases, 76(3), 431-435. https://doi.org/10.1053/j.ajkd.2020.05.004

Pronk, N. P., Anderson, L. H., Crain, A. L., Martinson, B. C., O'Connor, P. J., Sherwood, N. E., \& Whitebird, R. R. (2004). Meeting recommendations for multiple healthy lifestyle factors: prevalence, clustering, and predictors among adolescent, adult, and senior health plan members. American journal of preventive medicine, 27(2), 25-33. https://doi.org/10.1016/j.amepre.2004.04.022

Samb, B., Desai, N., Nishtar, S., Mendis, S., Bekedam, H., Wright, A., ... \& Etienne, C. (2010). Prevention and management of chronic disease: a litmus test for health-systems strengthening in low-income and middleincome countries. The Lancet, 376(9754), 1785-1797. https://doi.org/10.1016/S0140-6736(10)61353-0

Shingare, A., Bahadur, M. M., \& Raina, S. (2020). COVID-19 in recent kidney transplant recipients. American Journal of Transplantation, 20(11), 3206-3209.

Spoorthy, M. S., Pratapa, S. K., \& Mahant, S. (2020). Mental health problems faced by healthcare workers due to the COVID-19 pandemic-A review. Asian journal of psychiatry, 51, 102119. https://doi.org/10.1016/j.ajp.2020.102119

Toapanta, N., Torres, I. B., Sellarés, J., Chamoun, B., Serón, D., \& Moreso, F. (2021). Kidney transplantation and COVID-19 renal and patient prognosis. Clinical Kidney Journal, 14(Supplement_1), i21-i29.

Travis, P., Bennett, S., Haines, A., Pang, T., Bhutta, Z., Hyder, A. A., ... \& Evans, T. (2004). Overcoming healthsystems constraints to achieve the Millennium Development Goals. The Lancet,364(9437), 900-906. https://doi.org/10.1016/S0140-6736(04)16987-0

Waleka, E., Mackiewicz, M., Romanski, J., Dybko, A., Stojek, Z., \& Karbarz, M. (2020). Degradable nanohydrogel with high doxorubicin loadings exhibiting controlled drug release and decreased toxicity against healthy cells. International journal of pharmaceutics, 579, 119188. https://doi.org/10.1016/j.ijpharm.2020.119188

Widana, I.K., Sumetri, N.W., Sutapa, I.K., Suryasa, W. (2021). Anthropometric measures for better cardiovascular and musculoskeletal health. Computer Applications in Engineering Education, 29(3), 550561. https://doi.org/10.1002/cae.22202

Widjaja, G. (2021). Impact of human resource management on health workers during pandemics COVID-19: systematic review. International Journal of Health \& Medical Sciences, 4(1), 61-68.

Yarijani, Z. M., \& Najafi, H. (2021). Kidney injury in COVID-19 patients, drug development and their renal $\begin{array}{llll}\text { complications: } \quad \text { Review } & \text { study. Biomedicine \& } & \text { Pharmacotherapy, } & 111966 .\end{array}$ https://doi.org/10.1016/j.biopha.2021.111966

Rahkmonova, S., Khaytboyev, J., Zargarova, N., Otajonova, D., \& Kazakov, K. (2021). Kidney complications after COVID-19. International Journal of Health Sciences, 5(3), 393-402. https://doi.org/10.53730/ijhs.v5n3.1698 


\section{Biography of Authors}

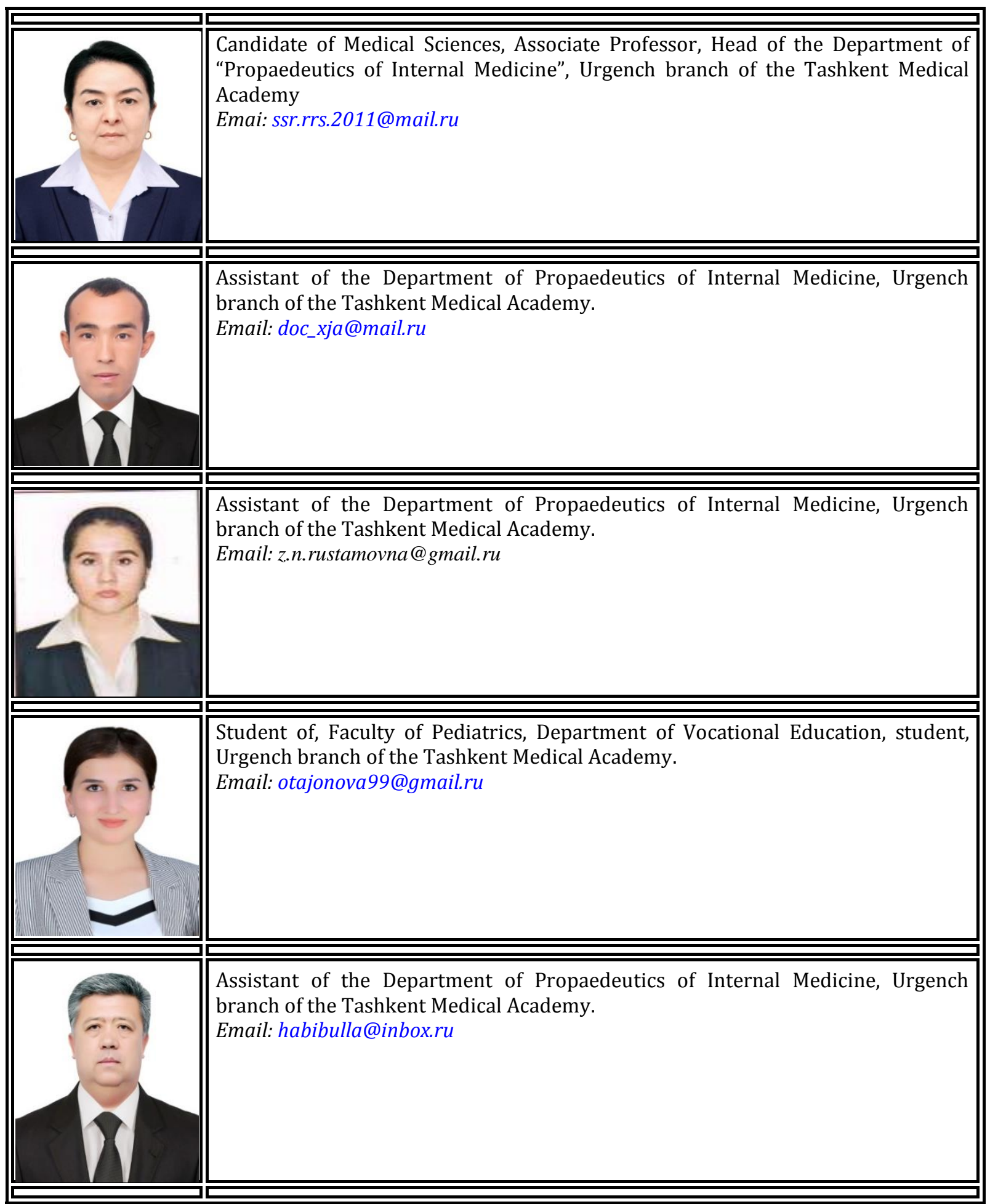

